\title{
DISCS AND BOUNDARY UNIQUENESS FOR PSH FUNCTIONS ON ALMOST COMPLEX MANIFOLD
}

\section{A. SUKHOV}

\begin{abstract}
This paper is inspired by the work by J.-P. Rosay (2010). In this work, there was sketched a proof of the fact that a totally real submanifold of dimension 2 can not be a pluripolar subset of an almost complex manifold of complex dimension 2 . In the present paper we prove a considerably more general result, which can be viewed as a boundary uniqueness theorem for plurisubharmonic functions. It states that a function plurisubharmonic in a wedge with a generic totally real edge is equal to $-\infty$ identically if it tends to $-\infty$ approaching the edge. Our proof is completely different from the argument by J.-P. Rosay. We develop a method based on construction of a suitable family of $J$ complex discs. The origin of this approach is due to the well-known work by S. Pinchuk (1974), where the case of the standard complex structure was settled. The required family of complex discs is obtained as a solution to a suitable integral equation generalizing the classical Bishop method. In the almost complex case this equation arises from the CauchyGreen type formula. We hope that the almost complex version of this construction presented here will have other applications.
\end{abstract}

Keywords:almost complex structure, plurisubharmonic function, complex disc, totally real manifold.

Mathematics Subject Classification: 32H02, 53C15

\section{INTRODUCTION}

This paper addresses some aspects of pluripotential theory on almost complex manifolds. In the general case of non-integrable almost complex structures, the development of this theory began recently and many natural questions remain open. Our main motivation arises from the paper by J.-P.Rosay [5], where several interesting properties of (non) pluripolar subsets of almost complex manifolds were established. In particular, he proved that a $J$-complex curve is a pluripolar set. On the other hand, [5] there was sketched a proof of the fact that a totally real submanifold of dimension 2 can not be a pluripolar subset of an almost complex manifold of complex dimension 2 (a hint for a higher dimension argument was also indicated without details).

In the present paper we prove a considerably more general result (Theorem 4.1), which can be viewed as a boundary uniqueness theorem for plurisubharmonic functions. It states that a function plurisubharmonic in a wedge with generic totally real edge is equal to $-\infty$ identically if it tends to $-\infty$ approaching the edge. Our proof is completely different from the argument of [5]. Our approach is based on construction of a suitable family of $J$-complex discs and is inspired by the well-known work [3]. We hope that the almost complex version of this construction

A. Sukhov, Discs and boundary uniqueness For PSh functions on an almost Complex manifold.

(c) Sukhov A. 2018.

Поступила 19 июня 2018 г.

The author is partially supported by Labex CEMPI. 
presented here will have other applications. Another approach to filling a wedge by discs is used in [6] and is based on stability results for the Riemann-Hilbert boundary value problem. The method of present paper is more direct and gives an additional information on geometry of the discs. The required family of complex discs is obtained as a solution to a suitable integral equation generalizing the classical Bishop method. In the almost complex case this equation arises from the Cauchy-Green type formula.

The paper is organized as follows. In Section 2 we recall basic properties of almost complex manifolds (see more in [1]). Section 3 contains the construction of Bishop type discs glued to a totally real manifold along a boundary arc. In section 4 we obtain the main result on boundary uniqueness for psh functions.

\section{Almost COMPLEX MANIFOLDS, DISCS AND PLURISUBHARMONIC FUNCTIONS}

Throughout this paper we assume that manifolds and structures are $C^{\infty}$ smooth although the main results remain true under considerably weaker regularity assumptions.

Let $M$ be a smooth manifold of dimension $2 n$. An almost complex structure $J$ on $M$ is a smooth map, which associates to every point $p \in M$ a linear isomorphism $J(p): T_{p} M \rightarrow$ $T_{p} M$ of the tangent space $T_{p} M$ satisfying $J(p)^{2}=-I$. Here $I$ denotes the identity map of $T_{p} M$. Thus, $J(p)$ is a linear complex structure on $T_{p} M$. A couple $(M, J)$ is called an almost complex manifold of complex dimension $n$. Note that every almost complex manifolds admits the canonical orientation.

The standard complex structure $J_{s t}=J_{s t}^{(2)}$ on $M=\mathbb{R}^{2}$ is given by the matrix

$$
J_{s t}=\left(\begin{array}{ll}
0 & -1 \\
1 & 0
\end{array}\right)
$$

in the canonical coordinates of $\mathbb{R}^{2}$. More generally, the standard complex structure $J_{s t}$ on $\mathbb{R}^{2 n}$ is represented by the block diagonal matrix $\operatorname{diag}\left(J_{s t}^{(2)}, \ldots, J_{s t}^{(2)}\right)$; we skip the notation of dimension because it will be clear from the context. As usually, setting $i v:=J v$ for $v \in \mathbb{R}^{2 n}$, we identify $\left(\mathbb{R}^{2 n}, J_{s t}\right)$ with $\mathbb{C}^{n}$ having $z=x+i y=x+J y$ for the standard complex coordinates $z=\left(z_{1}, \ldots, z_{n}\right) \in \mathbb{C}^{n}$.

Let $(M, J)$ and $\left(M^{\prime}, J^{\prime}\right)$ be smooth almost complex manifolds. A $C^{1}$-map $f: M^{\prime} \rightarrow M$ is called $\left(J^{\prime}, J\right)$-complex or $\left(J^{\prime}, J\right)$-holomorphic if it satisfies the Cauchy-Riemann equations

$$
d f \circ J^{\prime}=J \circ d f .
$$

Of course, a map $f: \mathbb{C}^{n} \rightarrow \mathbb{C}^{m}$ is $\left(J_{s t}, J_{s t}\right)$-holomorphic if and only if each component of $f$ is a usual holomorphic function.

Every almost complex manifold $(M, J)$ can be viewed locally as a unit ball $\mathbb{B}$ in $\mathbb{C}^{n}$ equipped with a small almost complex deformation of $J_{s t}$. Indeed, we have the following useful statement.

Lemma 2.1. Let $(M, J)$ be an almost complex manifold. Then for every point $p \in M$, every real $\alpha \geqslant 0$ and $\lambda_{0}>0$, there exist a neighborhood $U$ of $p$ and a coordinate diffeomorphism $z: U \rightarrow \mathbb{B}$ such that $z(p)=0, d z(p) \circ J(p) \circ d z^{-1}(0)=J_{\text {st }}$ and the direct image $z_{*}(J):=$ $d z \circ J \circ d z^{-1}$ satisfies $\left\|z_{*}(J)-J_{s t}\right\|_{C^{\alpha}(\overline{\mathbb{B}})} \leqslant \lambda_{0}$.

Доказательство. There exists a diffeomorphism $z$ from a neighborhood $U^{\prime}$ of $p \in M$ onto $\mathbb{B}$ satisfying $z(p)=0$ and $d z(p) \circ J(p) \circ d z^{-1}(0)=J_{s t}$. For $\lambda>0$, we consider the dilation $d_{\lambda}: t \mapsto \lambda^{-1} t$ in $\mathbb{R}^{2 n}$ and the composition $z_{\lambda}=d_{\lambda} \circ z$. Then $\lim _{\lambda \rightarrow 0}\left\|\left(z_{\lambda}\right)_{*}(J)-J_{s t}\right\|_{C^{\alpha}(\overline{\mathbb{B}})}=0$ for each real $\alpha \geqslant 0$. Setting $U=z_{\lambda}^{-1}(\mathbb{B})$ for $\lambda>0$ small enough, we obtain the desired statement.

Gromov's theory of pseudoholomorphic curves studies the property of solutions $f$ of $(2)$ in the special case, when $M^{\prime}$ has the complex dimension 1. These holomorphic maps are called 
$J$-complex (or $J$-holomorphic) curves. We use the notation $\mathbb{D}=\{\zeta \in \mathbb{C}:|\zeta|<1\}$ for the unit disc in $\mathbb{C}$ always assuming that it is equipped with the standard complex structure $J_{\text {st }}$. If in the above definition we have $M^{\prime}=\mathbb{D}$ we call such a map $f$ a $J$-complex disc or a pseudoholomorphic disc or just a holomorphic disc if $J$ is fixed. Similarly, if $M^{\prime}$ is the Riemann sphere, $f$ is called a $J$-complex sphere. These two classes of pseudo-holomorphic curves are particularly useful.

Let $(M, J)$ be an almost complex manifold and $E \subset M$ be a real submanifold of $M$. Suppose that a $J$-complex disc $f: \mathbb{D} \rightarrow M$ is continuous on $\overline{\mathbb{D}}$. With some abuse of terminology, we also call the image $f(\mathbb{D})$ simply by a disc and we call he image $f(b \mathbb{D})$ by the boundary of a disc. If $f(b \mathbb{D}) \subset E$, then we say that (the boundary of ) the disc $f$ is glued or attached to $E$ or simply that $f$ is attached to $E$. Sometimes such maps are called Bishop discs for $E$ and we employ this terminology. Of course, if $p$ is a point of $E$, then the constant map $f \equiv p$ always satisfies this definition.

In this paper we deal with a special class of real submanifolds. A submanifold $E$ of an almost complex $n$-dimensional $(M, J)$ is called totally real if at every point $p \in E$ the tangent space $T_{p} E$ contains no non-trivial complex vectors that is $T_{p} E \cap J T_{p} E=\{0\}$. This is a well-known fact that the (real) dimension of a totally real submanifold of $M$ does not exceed $n$; we will consider in this paper only $n$-dimensional totally real submanifolds that is the case of the maximal dimension.

A totally real manifold $E$ can be defined as

$$
E=\left\{p \in M: \rho_{j}(p)=0\right\}
$$

where $\rho_{j}: M \rightarrow \mathbb{R}$ are smooth functions with non-vanishing gradients. The condition of total reality means that for every $p \in E$ the $J$-complex linear parts of the differentials $d \rho_{j}$ are (complex) linearly independent.

A subdomain

$$
W=\left\{p \in M: \rho_{j}<0, j=1, \ldots, n\right\} .
$$

$\mathrm{t}$ is called the wedge with the edge $E$.

2.1. Cauchy-Riemann equations in coordinates. All our consideration are local, so (as above) we are in a neighborhood $\Omega$ of 0 in $\mathbb{C}^{n}$ with the standard complex coordinates $z=$ $\left(z_{1}, \ldots, z_{n}\right)$. We assume that $J$ is an almost complex structure defined on $\Omega$ and $J(0)=J_{s t}$. Let

$$
\begin{aligned}
& z: \mathbb{D} \rightarrow \Omega, \\
& z: \zeta \mapsto z(\zeta)
\end{aligned}
$$

be a $J$-complex disc. Setting $\zeta=\xi+i \eta$ we write (2) in the form $z_{\eta}=J(Z) Z_{\xi}$. This equation can be in turn written as

$$
z_{\bar{\zeta}}-A(z) \bar{z}_{\bar{\zeta}}=0, \quad \zeta \in \mathbb{D} .
$$

Here a smooth map $A: \Omega \rightarrow \operatorname{Mat}(n, \mathbb{C})$ is defined by the identity $L(z) v=A \bar{v}$ for any vector $v \in \mathbb{C}^{n}$ and $L$ is an $\mathbb{R}$-linear map defined by $L=\left(J_{s t}+J\right)^{-1}\left(J_{s t}-J\right)$. It is easy to check that the condition $J^{2}=-I d$ iq equivalent to the fact that $L$ is $\overline{\mathbb{C}}$-linear. The matrix $A(z)$ is called the complex matrix of $J$ in the local coordinates $z$. Locally the correspondence between $A$ and $J$ is one-to-one. Note that the condition $J(0)=J_{s t}$ means that $A(0)=0$.

If $Z^{\prime}$ are other local coordinates and $A^{\prime}$ is the corresponding complex matrix of $J^{\prime}$, then, as it is easy to check, we have the following transformation rule:

$$
A^{\prime}=\left(z_{z}^{\prime} A+z^{\prime} \bar{z}^{\prime}\right)\left(\bar{z}^{\prime}{ }_{\bar{z}}+\bar{z}_{z}^{\prime} A\right)^{-1}
$$

(see [7]). 
2.2. Plurisubharmonic functions on almost complex manifolds: background. Let $u$ be a real $C^{2}$ function on an open subset $\Omega$ of an almost complex manifold $(M, J)$. Denote by $J^{*} d u$ the differential form acting on a vector field $X$ by $J^{*} d u(X):=d u(J X)$. Given a point $p \in M$ and a tangent vector $V \in T_{p}(M)$, we consider a smooth vector field $X$ in a neighborhood of $p$ satisfying $X(p)=V$. The value of the complex Hessian ( or of the Levi form ) of $u$ with respect to $J$ at $p$ and $V$ is defined by $H(u)(p, V):=-\left(d J^{*} d u\right)_{p}(X, J X)$. This definition is independent of the choice of a vector field $X$. For instance, if $J=J_{s t}$ in $\mathbb{C}$, then $-d J^{*} d u=\Delta u d \xi \wedge d \eta$; here $\Delta$ denotes the Laplacian. In particular, $H_{J_{s t}}(u)\left(0, \frac{\partial}{\partial \xi}\right)=\Delta u(0)$.

Let us recall some basic properties of the complex Hessian (see, for instance, [2]).

Lemma 2.2. Consider a real function $u$ of class $C^{2}$ in a neighborhood of a point $p \in M$.

(i) Let $F:\left(M^{\prime}, J^{\prime}\right) \longrightarrow(M, J)$ be a $\left(J^{\prime}, J\right)$-holomorphic map, $F\left(p^{\prime}\right)=$ p. For each vector $V^{\prime} \in T_{p^{\prime}}\left(M^{\prime}\right)$ we have $H_{J^{\prime}}(u \circ F)\left(p^{\prime}, V^{\prime}\right)=H_{J}(u)\left(p, d F(p)\left(V^{\prime}\right)\right)$.

(ii) If $f: \mathbb{D} \longrightarrow M$ is a J-complex disc satisfying $f(0)=p$, and $d f(0)\left(\frac{\partial}{\partial \xi}\right)=V \in T_{p}(M)$, then $H_{J}(u)(p, V)=\Delta(u \circ f)(0)$.

Property (i) expresses the holomorphic invariance of the complex Hessian. Property (ii) is often useful in order to compute the complex Hessian on a given tangent vector $V$.

Let $\Omega$ be a domain $M$. An upper semicontinuous function $u: \Omega \rightarrow[-\infty,+\infty[$ on $(M, J)$ is $J$-plurisubharmonic (psh) if for every $J$-complex disc $f: \mathbb{D} \rightarrow \Omega$ the composition $u \circ f$ is a subharmonic function on $\mathbb{D}$. By Proposition 2.2 , a $C^{2}$ function $u$ is psh on $\Omega$ if and only if it has a positive semi-definite complex Hessian on $\Omega$ i.e. $H_{J}(u)(p, V) \geqslant 0$ for any $p \in \Omega$ and $V \in T_{p}(M)$. A real $C^{2}$ function $u: \Omega \rightarrow \mathbb{R}$ is called strictly $J$-psh on $\Omega$, if $H_{J}(u)(p, V)>0$ for each $p \in M$ and $V \in T_{p}(M) \backslash\{0\}$. Obviously, these notions are local: an upper semicontinuous (resp. of class $C^{2}$ ) function on $\Omega$ is $J$-psh (resp. strictly) on $\Omega$ if and only if it is $J$-psh (resp. strictly) in some open neighborhood of each point of $\Omega$.

A useful observation is that the Levi form of a function $r$ at a point $p$ in an almost complex manifold $(M, J)$ coincides with the Levi form with respect to the standard structure $J_{s t}$ of $\mathbb{R}^{2 n}$ if suitable local coordinates near $p$ are choosen. Let us explain how to construct these adapted coordinate systems.

As above, choosing local coordinates near $p$ we may identify a neighborhood of $p$ with a neighborhood of the origin and assume that $J$-holomorphic discs are solutions of (5).

Lemma 2.3. There exists a second order polynomial local diffeomorphism $\Phi$ fixing the origin and with linear part equal to the identity such that in the new coordinates the complex matrix A of $J$ (that is A from the equation (5)) satisfies

$$
A(0)=0, \quad A_{z}(0)=0
$$

Thus, by a suitable local change of coordinates one can remove the terms linear in $z$ in the matrix $A$. We stress that in general it is impossible to get rid of first order terms containing $\bar{z}$ since this would impose a restriction on the Nijenhuis tensor $J$ at the origin.

The author learned this result from unpublished E. Chirka's notes; see 2 for the proof. It was shown in [7] that in an almost complex manifold of (complex) dimension 2, a similar normalization is possible along a given embedded $J$-holomorphic disc.

As a typical consequence, we consider a totally real manifold $E$ defined by (3). Then the function $u=\sum_{j=1}^{n} \rho^{2}$ is strictly $J$-psh in a neighborhood of $E$. Indeed, it suffices to choose local coordinates near $p \in M$ according to Lemma 2.3. This reduces the verification to the well-known case of $J_{s t}$. 


\section{FILLING A WEDGE BY COMPLEX DISCS}

Here we construct a family of Bishop's type discs filling a wedge with a totally real edge. Each disc is glued to the edge $E$ along the upper semi-circle.

3.1. Case of the standard structure. First consider the model case $M=\mathbb{C}^{n}$ with $J=J_{s t}$ and $E=i \mathbb{R}^{n}=\left\{x_{j}=0, j=1, \ldots, n\right\}$. Denote by $W$ the wedge

$$
W=\left\{z=x+i y: x_{j}<0, j=1, \ldots, n\right\} .
$$

Let

$$
P_{0} \phi=\frac{1}{2 \pi i} \int_{b \mathbb{D}} \phi(\omega) \frac{d \omega}{\omega}
$$

denotes the average of a real function $\phi$ over $b \mathbb{D}$, and let also

$$
S \phi(\zeta)=\frac{1}{2 \pi i} \int_{b \mathbb{D}} \frac{\omega+\zeta}{\omega-\zeta} \phi(\omega) \frac{d \omega}{\omega}
$$

be the Schwarz integral. In terms of the Cauchy transform

$$
K f(\zeta)=\frac{1}{2 \pi i} \int_{b \mathbb{D}} \frac{f(\omega) d \omega}{\omega-\zeta}
$$

we have the following relation: $S=2 K-P_{0}$. As a consequence, the boundary properties of the Schwarz integral are the same as the classical properties of the Cauchy integral.

For a non-integer $r>1$ consider the Banach spaces $C^{r}(b \mathbb{D})$ and $C^{r}(\mathbb{D})$ (with the usual Hölder norm). This is classical that $K$ and $S$ are bounded linear mappings in these classes of functions. For real function $\phi \in C^{r}(b \mathbb{D})$ the Schwarz integral $S \phi$ is a function of class $C^{r}(\mathbb{D})$ holomorphic in $\mathbb{D}$; the trace of its real part on the boundary coincides with $\phi$ and its imaginary part vanishes at the origin. In particular, every holomorphic function $f \in C^{r}(\mathbb{D})$ satisfies the Schwarz formula $f=S \operatorname{Re} f+i P_{0} f$.

We are going to fill $W$ by complex discs glued to $i \mathbb{R}^{n}$ along the (closed) upper semi-circle $b \mathbb{D}^{+}=\left\{e^{i \theta}: \theta \in[0, \pi]\right\} ;$ let also $b \mathbb{D}^{-}:=b \mathbb{D} \backslash b \mathbb{D}^{+}$.

Fix a smooth real function $\phi: b \mathbb{D} \rightarrow \mathbb{R}$ such that $\phi \mid b \mathbb{D}^{+}=0$ and $\phi \mid b \mathbb{D}^{-}<0$.

Consider now a real $2 n$-parameter family of holomorphic discs $z^{0}=\left(z_{1}^{0}, \ldots, z_{n}^{0}\right): \mathbb{D} \rightarrow \mathbb{C}^{n}$ with components

$$
z_{j}^{0}(c, t)(\zeta)=x_{j}(\zeta)+i y_{j}(\zeta)=t_{j} S \phi(\zeta)+i c_{j}, j=1, \ldots, n
$$

Here $t_{j}>0$ and $c_{j} \in \mathbb{R}$ are parameters, $t=\left(t_{1}, \ldots, t,\right), c=\left(c_{1}, \ldots, c_{n}\right)$. The following properties of this family are obvious:

(i) for every $j$ one has $x_{j} \mid b \mathbb{D}^{+}=0$ and $x_{j}(\zeta)<0$ when $\zeta \in \mathbb{D}$ (by the maximum principle for harmonic functions).

(ii) the evaluation map $E v_{0}:(t, c) \mapsto z^{0}(c, t)(0)$ is one-to-one from $\left\{(c, t): c_{j}>0, t_{j} \in \mathbb{R}\right\}$ on $W$.

Our goal is to construct a local analog of this family in the general case.

3.2. General case. In order to write an integral equation defining a required family of discs, we need to employ an analog of the Schwarz formula and to choose suitable local coordinates. We proceed in several steps.

Step 1. Recall that for any complex function $f \in C^{r}(\mathbb{D})$ the Cauchy-Green transform is defined by 


$$
T f(\zeta)=\frac{1}{2 \pi i} \iint_{\mathbb{D}} \frac{f(\omega) d \omega \wedge d \bar{\omega}}{\omega-\zeta}
$$

This is a classical fact that $T: C^{r}(\mathbb{D}) \rightarrow C^{r+1}(\mathbb{D})$ is a bounded linear operator for every noninteger $r>0$. Furthermore, $(T f)_{\bar{\zeta}}=f$, i.e., $T$ solves the $\bar{\partial}$-equation in the unit disc. Recall also that the function $T f$ is holomorphic on $\mathbb{C} \backslash \overline{\mathbb{D}}$.

We have the following Green-Schwarz formula (see the proof, for example, in [7], although, of course, it can be found in the vaste list of classical works). Let $f=\phi+i \psi: \overline{\mathbb{D}} \rightarrow \mathbb{C}$ be a function in the class $C^{r}(\mathbb{D})$. Then for each $\zeta \in \overline{\mathbb{D}}$ one has

$$
f(\zeta)=S \phi(\zeta)+i P_{0} \psi+T f_{\bar{\zeta}}(\zeta)-\overline{T f_{\bar{\zeta}}(1 / \bar{\zeta})}
$$

Note that because of the "symmetrization" the real part of the sum of two terms containing $T$ vanishes on the unit circle. We also notice that the last term is holomorphic on $\mathbb{D}$.

Step 2. Now let $(M, J)$ be an almost complex manifold of complex dimension $n$ and $E$ be a totally real $n$-dimensional submanifold of $M$. We assume that $E$ and $W$ are given by (3) and (4), respectively.

First, according to Section 2 we choose local coordinates $z$ such that $p=0$ and the complex matrix $A$ of $J$ satisfies (7). For every $\tau>0$ small enough and $C>0$ big enough the functions

$$
\tilde{\rho}_{j}:=\rho_{j}-\tau \sum_{k \neq j} \rho_{k}+C \sum_{k=1}^{n} \rho_{k}^{2}
$$

are strictly $J$-psh in a neighborhood of the origin and the "truncated" wedge $W_{\tau}=\left\{\tilde{\rho}_{j}<0, j=\right.$ $1, \ldots, n\}$ is contained in $W$. After a $\mathbb{C}$-linear (with respect to $J_{s t}$ ) change of coordinates one can assume that $\tilde{\rho}_{j}=x_{j}+o(|z|)$. Consider now a local diffeomorphism

$$
\Phi: z=x_{j}+i y_{j} \mapsto z^{\prime}=x_{j}^{\prime}+i y_{j}^{\prime}=\tilde{\rho}_{j}+i y_{j}
$$

Then $\Phi(0)=0, d \Phi(0)=0$ and in the new coordinates $\tilde{\rho}_{j}=x_{j}$ (we drop the primes), $E=i \mathbb{R}^{n}$ and $W_{\tau}=\left\{x_{j}<0, j=1, \ldots, n\right\}$. We keep the notation $J$ for the direct image $\Phi_{*}(J)$. Then in the chosen coordinates the complex matrix still satisfies $A(0)=0$. Note also that the coordinate functions $x_{j}$ are strictly psh for $J$.

Finally, similarly to the proof of Lemma 2.1, for $\lambda>0$, we consider the isotropic dilations $d_{\lambda}: z \mapsto \lambda^{-1} z$ and the direct images $J_{\lambda}:=\left(d_{\lambda}\right)_{*}(J)$. Denote by $A(z, \lambda)$ the complex matrix of $J_{\lambda}$.

Step 3. For $\lambda>0$ small enough, we are looking for the solutions $z: \mathbb{D} \rightarrow \mathbb{C}^{n}$ to a Bishop's type integral equation

$$
z(\zeta)=h(z(\zeta), t, c, \lambda)
$$

with

$$
h(z(\zeta), t, c, \lambda)=t S \phi(\zeta)+i c+T A(z, \lambda) \bar{z}_{\bar{\zeta}}(\zeta)-\overline{T A(z, \lambda) \bar{z}_{\bar{\zeta}}(1 / \bar{\zeta})}
$$

where $t=\left(t_{1}, \ldots, t_{n}\right), t_{j}>0$ and $c \in \mathbb{R}^{n}$ are real parameters. Note that the first and the last terms in the right hand are holomorphic on $\mathbb{D}$. Therefore, any solution of $(10)$ satisfies the Cauchy-Riemann equations (5) i.e. is a $J$-complex disc. Furthemore, $x_{j}(\zeta)$ vanishes on $b \mathbb{D}^{+}$ (that is, $z\left(b \mathbb{D}^{+}\right) \subset E$ ) and is negative on $b \mathbb{D}^{-}$. Since the function $z \mapsto x_{j}$ is strictly $J$-psh, by the maximum principle the image $z(\overline{\mathbb{D}})$ is contained in $\bar{W}_{\tau}$.

The existence of solutions follows the implicit function theorem. Note that for $\lambda=0$, equation (10) admits solution (8). We consider the smooth map of Banach spaces

$$
\begin{aligned}
& H: C^{r}(\mathbb{D}) \times \mathbb{R}^{n} \times \mathbb{R}^{n} \times \mathbb{R} \longrightarrow C^{r}(\mathbb{D}), \\
& H:(z, c, t, \lambda) \mapsto h(z(\zeta), t, c, \lambda) .
\end{aligned}
$$


Obviously the partial derivative of $H$ in $z$ vanishes: $\left(D_{z} H\right)\left(z^{0}, c, t, 0\right)=0$, where $z^{0}$ is a disc given by ( 8). By the implicit function theorem, for every $(c, t, \lambda)$ close enough to the origin the equation $(10)$ admits a unique solution $z(c, t)(\zeta)$ of class $C^{r}(\mathbb{D})$ depending smoothly on parameters $(c, t)$ (as well as $\lambda$, of course).

Fixing $\lambda>0$, we consider the smooth evaluation map

$$
E v_{\lambda}:(c, t) \mapsto z(c, t)(0)
$$

which associates to each parameter the centre of the corresponding disc. Note that for $\lambda=0$ we obtain the linear mapping $E v_{0}(c, t)=(t S \phi(0)+i c)$ appeared already in the model case of the standard structure. More precisely, for every $\lambda>0$ and every $c \in \mathbb{R}^{n}$, the equation (10) has the unique constant solution $z=i c$ when $t=0$. Hence, $E v_{\lambda}$ admits the expansion $E v_{\lambda}(c, t)=E v_{0}(c, t)+o(|(c, t, \lambda)|)$.

Denote by $V$ the wedge $V=\left\{(c, t): c \in \mathbb{R}^{n}, t_{j}>0, j=1, \ldots, n\right\}$ with the edge $L=$ $\left\{(c, 0): c \in \mathbb{R}^{n}\right\}$. Then $E v_{0}(V)$ coincides with $W_{\tau}$ in a neighborhood of the origin. Furthermore, $E v_{\lambda}(L)=E$ for $\lambda>0$. Also, for $\alpha>0$ the "truncated" wedge $W_{\alpha}=\left\{x_{j}-\alpha \sum_{k \neq j} x_{k}<0\right\}$ with the edge $E$ is contained in $W_{\tau}$. The faces of the boundary of $W_{\alpha}$ are transverse to the face of $W_{\tau}$. Since this property is stable under small perturbations, we conclude that $W_{\alpha} \subset E v_{\lambda}(V)$ for all $\lambda$ small enough. In terms of the initial defining functions $\rho_{j}$ we have $\left\{z: \rho_{j}-\delta \sum_{k \neq j} \rho_{k}<0\right\} \subset W_{\varepsilon}$ when $\tau+\varepsilon<\delta$.

We summarize the said above in the following theorem.

Theorem 3.1. Let $E$ be a totally real submanifold (3) of an almost complex manifold $(M, J)$ and $W$ be a wedge (4). For every $\delta>0$ there exists a family of J-complex discs smoothly depending on $2 n$ real parameters with the following properties:

(i) the boundary of every disc is glued along $b \mathbb{D}^{+}$to $E$ and every disc is contained in $W$;

(ii) every point of the truncated wedge $\left\{z: \rho_{j}-\delta \sum_{k \neq j} \rho_{k}<0\right\}$ belongs to some disc.

Although we do not need this in the paper, note that every point of the wedge $E$ belongs to the boundary of some disc. Concerning regularity of manifolds and almost complex structures, it suffices to require the class $C^{\alpha}$ with real $\alpha>2$. We skip the details.

\section{BOUNDARY UNIQUENESS}

Now we can prove easily the main result of the present paper.

Theorem 4.1. Let E be a totally real submanifold (3) of an almost complex manifold $(M, J)$ and $W$ be a wedge (4). Assume that $u$ is a plurisubharmonic function in $W$ such that

$$
\lim \sup _{W \ni z \rightarrow E} u(z)=-\infty
$$

Then $u \equiv-\infty$.

Indeed, for every disc $f$ constructed in Theorem 3.1 the function $(u \circ f)(\zeta)$ is subharmonic on $\mathbb{D}$ and tends to $-\infty$ as $\zeta \rightarrow b \mathbb{D}^{+}$; therefore, $u \circ f \equiv-\infty$ (by classical properties of subharmonic functions, see [4]). Now by (ii) Theorem 3.1 we conclude that $u \equiv-\infty$.

Corollary 4.2. Let $E$ be a closed subset of an almost complex n-dimensional manifold $(M, J)$. Suppose that $E$ contains a germ of a totally real manifold of dimension $n$. Then $E$ is not a pluripolar set. 


\section{BIBLIOGRAPHY}

1. M.Audin, J.Lafontaine. Holomorphic curves in symplectic geometry. Progress in Mathematics. 117 Birkhauser, Basel (1994).

2. K. Diederich, A. Sukhov. Plurisubharmonic exhaustion functions and almost complex Stein structures // Mich. Math. J. 56:2, 331-355 (2008).

3. S. Pinchuk. A boundary uniqueness theorem for holomorphic functions of several complex variables // Mat. Zametki. 15:2, 205-212 (1974). [Math. Notes. 15:2, 116-120 (1974).]

4. Th. Ransford. Potential theory on the complex plane. Cambridge Univ. Press, Cambridge (1995).

5. J.-P. Rosay. Pluri-polarity in almost complex structures // Math. Z. 265:1, 133-149 (2010).

6. B. Coupet, H. Gaussier, A. Sukhov. Fefferman's mapping theorem on almost complex manifolds of complex dimension 2// Math. Z. 250:1, 59-90 (2005).

7. A. Sukhov, A.Tumanov. Filling hypersurfaces by discs in almost complex manifolds of dimension 2 // Indiana Univ. Math. J. 57:1, 509-544 (2008).

Alexandre Sukhov,

Université des Sciences et Technologies de Lille,

Laboratoire Paul Painlevé,

U.F.R. de Mathématiques,

59655 Villeneuve d'Ascq, Cedex, France

Institute of Mathematics,

Ufa Federal Research Center, RAS,

450008, Ufa, Russia

E-mail: sukhov@math.univ-lille1.fr 